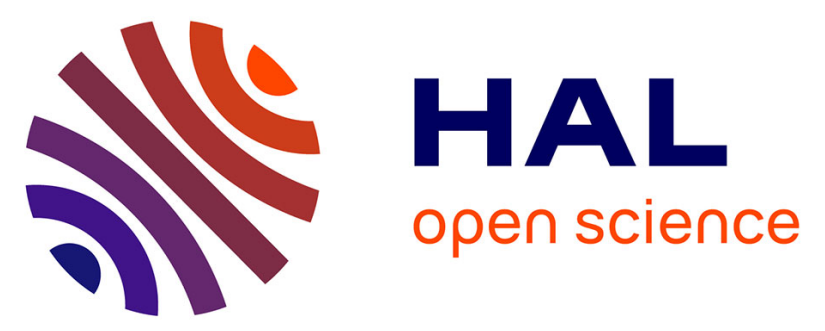

\title{
Relevance of cohort design for studying the prevalence of the ERG rearrangement in prostate cancer
}

Martin Braun, Veit Julian Scheble, Roopika Menon, Gregor Scharf, Theresia

Wilbertz, Karen Petersen, Christine Beschorner, Markus Reischl, Rainer

Kuefer, David Schilling, et al.

\section{To cite this version:}

Martin Braun, Veit Julian Scheble, Roopika Menon, Gregor Scharf, Theresia Wilbertz, et al.. Relevance of cohort design for studying the prevalence of the ERG rearrangement in prostate cancer. Histopathology, 2011, 58 (7), pp.1028. 10.1111/j.1365-2559.2011.03862.x . hal-00655187

\section{HAL Id: hal-00655187 https://hal.science/hal-00655187}

Submitted on 27 Dec 2011

HAL is a multi-disciplinary open access archive for the deposit and dissemination of scientific research documents, whether they are published or not. The documents may come from teaching and research institutions in France or abroad, or from public or private research centers.
L'archive ouverte pluridisciplinaire HAL, est destinée au dépôt et à la diffusion de documents scientifiques de niveau recherche, publiés ou non, émanant des établissements d'enseignement et de recherche français ou étrangers, des laboratoires publics ou privés. 


\section{Histopathology}

\section{Relevance of cohort design for studying the prevalence of the ERG rearrangement in prostate cancer}

\begin{tabular}{|c|c|}
\hline Journal: & Histopathology \\
\hline Manuscript ID: & HISTOP-06-10-0305.R2 \\
\hline Wiley - Manuscript type: & Original Article \\
\hline $\begin{array}{l}\text { Date Submitted by the } \\
\text { Author: }\end{array}$ & 20-Oct-2010 \\
\hline Complete List of Authors: & $\begin{array}{l}\text { Braun, Martin; University Hospital of Tuebingen, Institute of } \\
\text { Pathology } \\
\text { Scheble, Veit; University Hospital of Tuebingen, Institute of } \\
\text { Pathology } \\
\text { Menon, Roopika; University Hospital of Tuebingen, Institut of } \\
\text { Pathology } \\
\text { Scharf, Gregor; University Hospital of Tuebingen, Institute of } \\
\text { Pathology } \\
\text { Wilbertz, Theresia; University Hospital of Tuebingen, Institute of } \\
\text { Pathology } \\
\text { Petersen, Karen; University Hospital Tuebingen, Institute of } \\
\text { Pathology } \\
\text { Beschorner, Christine; University Hospital of Tuebingen, Institute of } \\
\text { Pathology } \\
\text { Reischl, Markus; Research Center Karlsruhe, Institute for Applied } \\
\text { Informatics } \\
\text { Kuefer, Rainer; University Hospital of Ulm, Department of Urology } \\
\text { Schilling, David; University Hospital of Tuebingen, Department of } \\
\text { Urology } \\
\text { Stenzl, Arnulf; University Hospital of Tuebingen, Department of } \\
\text { Urology } \\
\text { Kristiansen, Glen; University of Zurich, Institute of Surgical } \\
\text { Pathology } \\
\text { Rubin, Mark; Weill Cornell Medical College, Department of } \\
\text { Pathology and Laboratory Medicine } \\
\text { Fend, Falko; University Hospital of Tuebingen, Institute of } \\
\text { Pathology } \\
\text { Perner, Sven; University Hospital of Tuebingen, Institute of } \\
\text { Pathology }\end{array}$ \\
\hline Keywords: & $\begin{array}{l}\text { ERG rearrangement, TMPRSS2-ERG, gene fusions, prostate cancer, } \\
\text { transition zone }\end{array}$ \\
\hline
\end{tabular}




\section{SCHOLARONE ${ }^{m}$ \\ Manuscripts}


Relevance of cohort design for studying the prevalence of the ERG rearrangement in prostate cancer

Martin Braun ${ }^{1}$, Veit J. Scheble ${ }^{1}$, Roopika Menon ${ }^{1}$, Gregor Scharf ${ }^{1}$, Theresia Wilbertz ${ }^{1}$, Karen Petersen ${ }^{1}$, Christine Beschorner ${ }^{1}$, Markus Reischl ${ }^{5}$, Rainer Kuefer $^{6}$, David Schilling ${ }^{2}$, Arnulf Stenzl ${ }^{2}$, Glen Kristiansen ${ }^{3}$, Mark A. Rubin ${ }^{4}$, Falko Fend ${ }^{1}$, Sven Perner ${ }^{1}$

${ }^{1}$ Institute of Pathology and ${ }^{2}$ Department of Urology; Comprehensive Cancer Center, University Hospital of Tuebingen, Tuebingen, Germany

${ }^{3}$ Institute of Surgical Pathology; University Hospital Zurich, Zurich, Switzerland

${ }^{4}$ Department of Pathology and Laboratory Medicine, Weill Cornell Medical College, New York, NY, USA

${ }^{5}$ Institute for Applied Informatics, Research Center Karlsruhe

${ }^{6}$ Department of Urology, University Hospital of UIm, UIm, Germany

Corresponding author:

Sven Perner, MD

Institute of Pathology

University Hospital of Bonn

Sigmund-Freud-Str. 25

D-51327 Bonn

sven.perner1972@gmail.com

phone: $\quad+4915158233448$ 


\section{Acknowledgements:}

This work was supported by a grant of the German Research Foundation (Deutsche Forschungsgemeinschaft, DFG, Emmy-Noether-Program, PE1179/2-1) and the University Hospital of Tuebingen (fortuene Program, No. $1809-1-0)$ to S.P.

\section{Conflict of Interest}

The Brigham and Women's Hospital and the University of Michigan have filed a patent on ETS gene rearrangements in prostate cancer, on which S.P. and M.A.R are co-inventors and the diagnostic field of use has been licensed to GenProbe Inc. GenProbe has not played a role in the design and conduct of the study, nor in the collection, analysis or interpretation of the data and no involvement in the preparation, review or approval of the manuscript. 


\section{Abstract:}

Aims: $E R G$ rearrangements, mostly resulting in TMPRSS2-ERG fusions, are frequent alterations in prostate cancer ( $\mathrm{PCa}$ ) with a prevalence ranging from $15-78 \%$. Since the reason for this variability is unknown, we investigated the ERG rearrangement prevalence depending on cohort design.

Methods and Results: We assessed three well-defined cohorts for ERG rearrangements using FISH. The first cohort comprises 119 prostatectomy specimens. The second and third cohort includes incidentally diagnosed PCa (71 cystoprostatectomy specimens, and 105 TUR-P specimens). 70/119 (59\%) cases of the prostatectomy cohort harbored ERG rearrangements. Considering zonal origin, 2/11 (18\%) transition zone (TZ) foci and 75/145 (52\%) peripheral zone (PZ) foci exhibited ERG rearrangements. Within the cystoprostatectomies, 24/71 (34\%) cases revealed ERG rearrangements. Considering zonal origin, 2/9 (22\%) TZ foci and 26/86 (30\%) PZ foci harbored ERG rearrangements. PCa incidentally identified by TUR-P revealed ERG rearrangements in 31/105 (29\%) cases.

Conclusions: $E R G$ rearrangements also occur in TZ PCa, although at a lower prevalence as compared to PZ PCa. We confirmed that approximately half of all prostatectomies harbor ERG rearrangements. However, the prevalence in incidentally diagnosed PCa cohorts was 
significantly lower, even if considering multifocality. Consequently, zonal origin and cohort design are key for studying clinical implications of ERG rearrangements.

Running title: $E R G$ rearrangements in different cohorts

Key words: $E R G$ rearrangement, TMPRSS2-ERG, gene fusions, prostate cancer, transition zone 


\section{Introduction:}

The $E R G$ rearrangement is a common genetic alteration in prostate cancer (PCa). ${ }^{1}$ With a total number of over 2200 cases studied, the body of literature indicates a so far inexplicable and significant variability in the prevalence of this alteration ranging from $15 \%$ to $78 \%$, with the majority of studies consistently reporting a frequency of $50 \%$ in $\mathrm{PCa}{ }^{2}$ Moreover, a potential association between ERG rearrangement status and clinical outcome is still controversial. It remains elusive if $E R G$ rearrangement prevalence, clinical outcome and other aspects such as cohort design interfere with each other. Consequently, the aim of our study was to elucidate the reason for the reported variability in the $E R G$ rearrangement prevalence. Another aim was to prove the finding by Guo et al., that the $E R G$ rearrangement does not occur in $\mathrm{PCa}$ originating from the prostatic transition zone. ${ }^{3}$

\section{Materials and methods:}

\section{Cohorts:}

We assessed three different PCa cohorts for their ERG rearrangement status using an ERG break-apart FISH assay.

In detail, the first cohort contains tumor material from 119 Middle European partially PSA-screened patients who consecutively underwent 
radical prostatectomy as single therapeutic modality at the University Hospital of Tuebingen. For Germany, there are hardly any robust data available on how many men are undergoing PSA screening periodically. According to an estimation published by Sieverding et al. ${ }^{4}$ approximately $20 \%$ of men older than 45 years are PSA screened annually, approximately $33 \%$ of men are PSA screened regularly in a two to three years period and approximately $75 \%$ of patients with PCa who underwent prostatectomy have been diagnosed by a single event of an increased PSA level. According to these data, this prostatectomy cohort is best described as "partially PSA screened".

In the partially PSA-screened PCa cohort, multifocality and location (prostatic peripheral zone (PZ) versus prostatic transition zone (TZ)) of the tumor foci was taken into account. Prior to tissue microarray (TMA) construction, geographically discrete $\mathrm{PCa}$ foci were identified on corresponding H\&E slides. Ipsilateral tumor foci were defined as geographically distinct if they were separated by a minimum of $3 \mathrm{~mm}$ from the nearest tumor focus in any single section or by a minimum of 4 $\mathrm{mm}$ from the closest focus on the adjacent section above or below. ${ }^{5-8}$ Each distinct PCa focus was represented by three cores on a TMA. In case of multifocality, the tumour focus with the largest tumour diameter was considered as the primary focus. Secondary and tertiary tumour foci had the second largest and third largest tumour diameter, respectively. The zonal origin of each PCa focus was determined according to McNeal's zonal anatomy model. All PCa specimens were completely embedded in 
standard cassettes according to the CAP Cancer Committee protocol guidelines (see www.cap.org).

The second cohort includes PCa tissue from 71 Middle European urinary bladder cancer patients who consecutively underwent cystoprostatectomy at the University Hospital of Ulm, additionally suffering from incidentally diagnosed PCa. Prior to TMA construction, geographically discrete PCa foci were identified on H\&E slides. Considering PCa multifocality and focus location, each geographically discrete PCa focus is represented by three cores on a TMA, as described above. Prior to TMA construction, discrete tumour foci were categorized according to decreasing tumour diameter.

The third cohort comprises tumour material from 105 Middle European PCa patients consecutively incidentally diagnosed by trans-urethral resection of the prostate (TUR-P) at the University Hospital of Tuebingen. In order to take potential PCa multifocality into account, tumour foci with different Gleason patterns were preselected on corresponding H\&E slides. Depending on the tumour volume and the number of assumed distinct foci, two to nine tumour cores per case were captured for TMA construction. All TMAs were constructed as described earlier, ${ }^{1,9}$ each tumour focus of the three cohorts was centrally reassessed by one pathologist (S.P.) according to the revised Gleason grading system.

\section{ERG break-apart FISH assay}

We used a fluorescence in-situ hybridization (FISH) assay to detect the ERG rearrangement at the chromosomal level on formalin-fixed paraffin- 
embedded specimen. We performed a split-signal-approach, with two probes spanning the ERG locus as described earlier. ${ }^{9,10}$ Deparaffinized sections were pre-treated with a $100 \mathrm{mM}$ Tris and $50 \mathrm{mM}$ EDTA solution at $92.8 \mathrm{C}^{\circ}$ for $15 \mathrm{~min}$. and digested with Digest-All III (dilution $1: 2$ ) at $37^{\circ} \mathrm{C}$ for $22 \mathrm{~min}$.; $E R G$ FISH probes were denatured at $73^{\circ} \mathrm{C}$ for $5 \mathrm{~min}$. and immediately placed on ice. Subsequently, the tissue sections and ERG FISH probes were co-denatured at $94^{\circ} \mathrm{C}$ for $3 \mathrm{~min}$. and hybridised overnight at $37^{\circ} \mathrm{C}$. We used BAC clones RP11-24A11 for centromeric labelling with biotin and RP11-372017 for telomeric labelling with digoxigenin. Posthybridisation washing was done with $2 \times \mathrm{SSC}$ at $75^{\circ} \mathrm{C}$ for 7 min., and the fluorescence detection was carried out using streptavidinAlexa-594 conjugates (dilution 1:400) and anti-digoxigenin-FITC (dilution 1:400). Slides were then counterstained with 4',6-Diamidin-2' phenylindoldihydrochlorid (DAPI) and mounted.

The samples were analysed under an $63 x$ oil immersion objective using a fluorescence microscope (Zeiss, Jena, Germany) equipped with appropriate filters, a charge-coupled device camera and the FISH imaging and capturing software Metafer 4 (Metasystems, Altlussheim, Germany) . The evaluation of the tests was done independently by two experienced observers (M.B. and V.S.). At least 100 nuclei per case were evaluated and assessed as earlier described. ${ }^{11,12}$

This FISH assay allows for $E R G$ rearrangement status (i.e. rearrangement versus no rearrangement of $E R G$ ) assessment. The assay is also capable of differentiating between two different mechanisms of ERG 
rearrangement ${ }^{11,12}$. These two mechanisms are $E R G$ rearrangement through insertion and ERG rearrangement through deletion of DNA between TMPRSS2 and ERG loci (interstitial deletion). A nucleus without an $E R G$ rearrangement demonstrates two pairs of juxtaposed red and green signals (mostly forming 2 yellow signals). A nucleus with an ERG rearrangement through insertion shows the split of a signal pair resulting in a single red and single green signal for the rearranged $E R G$ allele and a still juxtaposed (yellow) signal pair for the non-rearranged $E R G$ allele in each nucleus. A nucleus with an ERG rearrangement through deletion shows one juxtaposed red-green signal pair (yellow) for the nonrearranged allele and a single red signal for the allele involved in the rearrangement. See Fig. 1 for details.

\section{Statistics}

In order to assess whether the $E R G$ rearrangement prevalence is different in the three PCa cohorts, we performed the Pearson's $\chi^{2}$-test (two-tailed). To correlate Gleason score and $E R G$ rearrangement status, we performed a t-test (two-tailed). A p-value lower than 0.05 was considered statistically significant. 


\section{Results:}

As an internal reference cohort, we assessed a partially PSA-screened prostatectomy cohort and observed the ERG rearrangement in 70/119 (58.9\%) of cases and in a total of $77 / 156$ (49.4\%) foci (Fig. 2A). Of these, $26 / 77$ foci $(33.8 \%$ ) harbored the ERG rearrangement through insertion, whereas $51 / 77$ foci $(66.2 \%)$ harbored the ERG rearrangement through deletion. Multifocal tumours were present in 41 cases. Of note, $17 / 119(14.3 \%)$ cases showed interfocal heterogeneity with regard to the $E R G$ rearrangement status. Six cases (Fig. 3A, cases \#2, \#3, \#5, \#6, \#8, \#17) exhibited interfocal ERG rearrangement heterogeneity with regard to the rearrangement mechanism (i.e. ERG rearrangement through deletion versus ERG rearrangement through insertion). When differentiating between tumour foci of the $\mathrm{TZ}$ and $\mathrm{PZ}$, we identified the ERG rearrangement in $2 / 11(18.1 \%)$ of the TZ foci and $75 / 145(51.7 \%)$ of the $\mathrm{PZ}$ foci. The number of cases with ERG rearranged PZ and TZ tumour foci were $68(57.3 \%)$ and $2(1.6 \%)$, respectively. Considering tumour diameters, the primary, secondary and tertiary tumour foci showed the ERG rearrangement in $62 / 109$ (56.9\%) foci, $13 / 41$ (31.7\%) foci and $2 / 6$ (33.3\%) foci, respectively.

In the incidentally diagnosed PCa cohorts (i.e. the cystoprostatectomy and TUR-P cohort), we detected the ERG rearrangement in 55/176 (31.2\%) of cases. In detail, the cystoprostatectomy PCa cohort exhibited the ERG rearrangement in $24 / 71(33.8 \%)$ cases and in $28 / 95(29.5 \%)$ tumour foci 
(Fig. 2B). Within this cohort, $9 / 24(37.5 \%)$ cases harbored the $E R G$ rearrangement through deletion and $15 / 24(62.5 \%)$ cases harbored the $E R G$ rearrangement through insertion. Multifocal tumours were present in $21(29.6 \%)$ cases. In these 21 multifocal tumours, we detected interfocal $E R G$ heterogeneity in five cases. Of these, four cases (Fig. 3B, cases \#14) revealed interfocal $E R G$ heterogeneity with regard to $E R G$ rearrangement status (i.e. $E R G$ rearrangement versus no $E R G$ rearrangement) and one case (Fig. 3B, cases \#5) showed interfocal ERG rearrangement heterogeneity with regard to the rearrangement mechanism (i.e. $E R G$ rearrangement through deletion versus $E R G$ rearrangement through insertion). When differentiating between TZ and PZ tumour foci, 2/9 (22.2\%) TZ foci and 26/86 (30.2\%) PZ foci exhibited the ERG rearrangement. The number of cases with ERG rearranged $\mathrm{PZ}$ and TZ foci were $22(30.8 \%)$ and $2(2.8 \%)$, respectively. Considering approximate tumour diameter, the primary, secondary and tertiary tumour foci showed the ERG rearrangement in $24 / 69$ (34.8\%) foci, $4 / 21$ $(19.0 \%)$ foci, and $1 / 5(20.0 \%)$ foci, respectively.

The TUR-P PCa cohort revealed the ERG rearrangement in 31/105 $(29.5 \%)$ cases with $25 / 31(80.6 \%)$ cases harboring the ERG rearrangement through deletion and 6/31 (19.4\%) through insertion (Fig. 2C). Since we took multifocality into account and therefore selected two to nine randomly sampled cores per case depending on the tumour volume, we found that 9/105 (8.6\%) cases (Fig. 3C, case \#1-9) revealed heterogeneity with regard to the ERG rearrangement status between the 
different TMA cores of an individual case (i.e. ERG rearrangement versus no ERG rearrangement). One case (Fig. 3C, case \#10) exhibited heterogeneity with regard to ERG rearrangement mechanism between different cores of an individual case (i.e. $E R G$ rearrangement through deletion versus $E R G$ rearrangement through insertion).

The overall prevalence of the $E R G$ rearrangement was significantly higher in the prostatectomy cohort as compared to each of the incidentally diagnosed cohorts (i.e. $\mathrm{p}<0.001$ for both, the cystoprostatectomy and TUR-P cohort). As expected, there was no significant difference with regard to the overall ERG rearrangement prevalence between the two incidentally diagnosed PCa cohorts. For the prostatectomy cohort, the ERG rearrangement prevalence was significantly lower in tumour foci originating from the $T Z$ as compared to $P Z$ tumour foci $(p<0.05)$. The prevalence of ERG rearranged TZ tumours within the cystoprostatectomy was lower as compared to PZ tumours. However, this finding did not reach statistical significance in our study. Of note, none of the cases with an $E R G$ rearranged $\mathrm{TZ}$ focus simultaneously had an $E R G$ rearranged $\mathrm{PZ}$ focus. Also, there was no case with two independent ERG rearranged TZ foci. However, due to the relatively low number of $T Z$ foci available within the two cohorts, we can not exclude that such constellations appear in other studies.

Considering tumour diameters, the ERG rearrangement prevalence of primary tumour foci (i.e. largest tumour focus) was higher as compared to the secondary and tertiary tumour foci. This prevalence difference was 
highly significant for the prostatectomy cohort (primary vs. secondary foci, $p<0.01$ ), but not significant for the cystoprostatectomy cohort. Additionally, even though tertiary tumour foci harboured the ERG rearrangement in a lower number as compared to primary foci, this difference was not statistically significant. Within the incidentally diagnosed TUR-P cohort, the ERG rearrangement was significantly associated with higher Gleason score $(p<0.05)$. However, no significant correlation could be found for each other constellation or cohort. Table 1 summarises these results.

\section{Discussion:}

The $E R G$ rearrangement is a common, and highly specific genetic event in PCa and most often results in a TMPRSS2-ERG gene fusion. ${ }^{1,2}$ However, in a smaller fraction the $E R G$ rearrangement results in rarer fusions such as SLC45A3-ERG ${ }^{13}$ or NDRG1-ERG. ${ }^{14}$ The ERG gene rearrangement as determined by FISH includes these three gene fusions. The prevalence ranges from $15 \%$ to $78 \%$ with the majority of studies consistently reporting a prevalence of $50 \%$ in $\mathrm{PCa} .{ }^{2}$ The focus of the current study was to explore why the prevalence varies significantly in the body of literature. To address this, we assessed the $E R G$ rearrangement prevalence in three distinct and population-based PCa cohorts. We categorized these cohorts 
according to how cases were identified clinically (i.e. partially PSAscreened prostatectomy, cystoprostatectomy, and TUR-P). Of note, an additional meta-analysis of previously published studies was not considered to be revealing. This is because most studies do not provide comprehensive clinicopathological data on their assessed cohorts. Moreover, they are derived from different populations, and normally differ in inter-institutional diagnostic and therapeutic modalities as well as in their experimental design and evaluation. The few commonly provided information in the studies published so far were: methods on how the cancer was diagnosed (i.e. incidentally, by PSA-screening and/or biopsy), ethnicity, and ERG rearrangement detection method (i.e. FISH or PCR). Given that, in our aim to identify ERG prevalence affecting factors, we focussed our assessment on these manifested points. In addition we took multifocality and tumour localization into account. These points were not available in most of the previous studies, but for our cohorts.

We could confirm that the $E R G$ rearrangement appears in approximately $50 \%$ of all cases in PSA-screened prostatectomy cohorts ${ }^{2,13,15}$ and at a significantly lower frequency $(<35 \%, \mathrm{p}<0.05)$ in incidentally diagnosed PCa cohorts. ${ }^{16,17}$ By this means, our data also confirm the assumption that the $E R G$ rearrangement prevalence is unlikely to differ between Caucasian populations, since Kumar-Sinha et al. reviewed on similar prevalences among 25 North-American and Western-European PCa cohorts. ${ }^{18}$ Of note, a recently published report by Mao et al. strongly suggests, that this does not apply for Non-Caucasian, such as Chinese populations. ${ }^{19}$ 
PCa is a multifocal disease in a multitude of cases. ${ }^{5-8}$ In our PCa cohorts, we considered multifocality. The fact that the ERG rearrangement prevalence in each of our cohorts is found at the upper range of comparable cohorts is most likely due to our strategy of taking multifocality into account. This is further supported by the observation, that in both, the prostatectomy and the TUR-P cohort, the ERG rearrangement prevalence of all tumor foci within one cohort $(49 \%$ and $29 \%$, respectively) is lower than the ERG rearrangement prevalence of all cases (59\% and 34\%, respectively). Most former studies have not addressed multifocality of localized PCa and only assessed one tumour focus per case, mainly the index tumour focus. We show evidence that if all tumour foci within a prostate gland are assessed for the ERG rearrangement status, the overall ERG rearrangement frequency increases.

We applied the so far most commonly used and reliable method of $E R G$ rearrangement assessment, i.e. the FISH-based break-apart assay as originally described. ${ }^{10,11}$ Unlike PCR-based identification methods, this assay allows for simultaneous detection of all gene rearrangements involving ERG (i.e. TMPRSS2-ERG, SLC45A-ERG, and NDRG-ERG), ${ }^{13,14}$ as well as the two different rearrangement mechanisms (i.e. ERG rearrangement through deletion and ERG rearrangement through insertion). ${ }^{11,12}$ The $E R G$ rearrangement prevalence in each of our cohort is similar to the frequencies of comparable cohorts. Consequently, we assume that the reported variability in the $E R G$ rearrangement frequencies 
is not affected by minor variations in the design and performance of the FISH assay or by matter of assay interpretation.

In contrast to a recent report by Guo et al., we could identify the ERG rearrangement in two PCa foci of the prostatic TZ in our cystoprostatectomy cohort and in two PCa foci of the TZ in our prostatectomy cohort (Fig. 4). ${ }^{3}$ TZ PCa has been shown to have a differential gene expression profile as compared to PZ tumours. ${ }^{20}$ Additionally, several studies suggested that a subset of TZ tumour foci is associated with more favourable pathological features than their PZ counterparts. ${ }^{21-25}$ Compared to PZ tumour foci, transition zone PCa are less likely to extend through the capsule ${ }^{24-26}$, have a higher rate of biochemical PSA cure ${ }^{26}$ and are better differentiated 25,27 - although they are often characterised by higher preoperative PSA levels and a larger tumour volume. ${ }^{21-23}$ In both, the prostatectomy and the cystoprostatectomy cohort, only the minority of TZ tumor foci $(18.1 \%$ and $22.2 \%$, respectively) exhibited an $E R G$ rearrangement. In the prostatectomy cohort, the prevalence of ERG rearrangements in this zone was significantly lower as compared to $P Z$ tumour foci $(p<0.01,18.1 \%$ vs. $51.7 \%$ ). Of note, the low prevalence of ERG rearranged TZ tumours has already been described in a recently published study by Falzarano et al. ${ }^{28}$ To conclude, the low frequency of ERG rearrangements in TZ tumours strengthens the assumption, that TZ tumours are distinct lesions with regard to tumor biology and molecular behaviour. Of interest, none of the cases with an ERG rearranged TZ focus simultaneously had an ERG 
rearranged $\mathrm{PZ}$ focus. Given the low frequency of $E R G$ rearrangements in TZ tumours, the discrepancy of the prevalence among different cohorts types (i.e. incidentally diagnosed and PSA-screened) might be influenced by zonal tumour origin. Mainly, since it is supposed that TURP sampled cohorts predominantly include TZ tumours, this might explain the prevalence difference to PSA-screened cohorts. Controversially, this hypothesis seems not to apply for the incidentally diagnosed cystoprostatectomy cohort, where the overall prevalence is equally low, even though tumour foci predominantly derive from the PZ. Also of interest regarding that issue, PZ tumour foci within the cystoprostatectomy cohort showed the ERG rearrangement in a comparable low prevelance as TZ foci (30\% vs. $22 \%$ ).

In our TUR-P cohort, we found a significant association between the presence of ERG rearrangements and higher Gleason scores, whereas there was no such association in our prostatectomy and cystoprostatectomy cohorts. Considering tumour diameters of the prostatectomy cohort, the ERG rearrangement prevalence of primary tumour foci (i.e. largest tumour focus) was significantly higher as compared to secondary tumour foci. In the cystoprostatectomy cohort, a comparable difference was observed, but the difference did not reach statistical significance. These controversial findings reflect the data in the body of literature. I.e., many former studies reported an association of $E R G$ rearrangement with non-favourable pathologic factors, such as higher Gleason score, increased stage, volume or early biochemical recurrence. ${ }^{2}$ 
11, 29-33 On the other hand, some studies reported an association of ERG rearrangement with parameters of more favourable outcome. ${ }^{34-35}$ Furthermore, a set of studies without any such association were reported as well. ${ }^{19,36-40}$ Still, in our hands, the cohorts with the naturally more favourable clinical courses (i.e. incidentally diagnosed prostate cancers) had a significantly lower ERG prevalence (approx. 30\%), as compared to the PSA-screened prostatectomy cohort (approx. 60\%), which might indirectly suggest an association with aggressiveness of the disease.

In conclusion, our findings support the view that the underlying molecular genetics of TZ tumours are different than these of PZ tumours. Furthermore, we could provide evidence that cohort design (i.e. method on how the diagnosis is based), zonal origin (i.e. TZ or PZ), and considering multifocality might have an impact on the $E R G$ rearrangement prevalence. Regarding cohort design, our findings would also apply for other studies, since the ERG prevalence of comparable cohort types are at similar levels. ${ }^{2,16-18}$ Regarding zonal origin, we confirmed a just recently published work on the low ERG prevalence of TZ tumour foci. ${ }^{28}$ Based on our findings, we cautiously assume that either the proportion of PZ/TZ tumours within a cohort and/or the proportion of aggressive/indolent cancers within the cohorts might be responsible for these differences. Yet, we more directly demonstrate that respecting cohort design as well as considering multifocality and zonal origin of the tumor is key for studying ERG rearrangements in PCa. The approach of our study may also apply to 
establish a better understanding of other PCa related molecular alterations and so far suggested biomarkers. 


\section{Figure Legends:}

\section{Figure 1:}

Detection of the $E R G$ rearrangement.

Schematic displaying the dual colour probe $E R G$ break-apart Fluorescence in-situ Hybridization (FISH) assay as used for the indirect detection of ERG Rearrangements. Red-labelled centromeric and the green-labelled telomeric probes span the $E R G$ locus on chromosomes 21. If a break-apart occurs, the green signal is either lost (ERG rearrangement through deletion) or translocated (ERG rearrangement through insertion). An ERG break-apart as determined by FISH accounts for a fusion of ERG with TMPRSS2, SLC45A or NDRG.

A: Wild type $E R G$ rearrangement status. Metaphase chromosomes 21 with dual colour probes spanning the centromeric and telomeric region of the $E R G$ locus. Interphase nucleus of a peripheral zone prostate cancer cell (below) shows one pairs of adjacent red and green signals with yellow signal overlapping, depicting a wild type ERG rearrangement status.

B: ERG rearrangement through deletion. One chromosome 21 lacks the telomeric probe spanning the ERG locus. Interphase nucleus of a transitional zone prostate cancer cell (below) shows two adjacent red and green signals, as well as one single red signal, depicting a heterozygous $E R G$ rearrangement through deletion. 
C: $E R G$ rearrangement through insertion. The $5^{\prime}$ telomeric $E R G$-spanning probe of one chromosome 21 is translocated to another chromosome. Interphase nucleus of a peripheral zone prostate cancer cell (below) shows two adjacent red and green signals, as well as one separate red and green signal, depicting a heterozygous ERG rearrangement through insertion.

\section{Figure 2:}

$E R G$ rearrangement prevalence of two incidentally diagnosed PCa cohorts and one PSA-screened prostatectomy cohort. Each core/focus of an individual case was independently assessed for the ERG rearrangement status.

A: Pie chart displaying the $E R G$ rearrangement status of the prostatectomy PCa cohort.

B: Pie chart displaying the $E R G$ rearrangement status of the cystoprostatectomy PCa cohort.

C: Pie chart displaying the ERG rearrangement status of the TUR-P cohort.

\section{Figure 3:}


$E R G$ rearrangement heterogeneity of two incidentally diagnosed PCa cohorts and one PSA-screened prostatectomy cohort. Each core/focus of an individual case was independently assessed for the $E R G$ rearrangement status.

A: Summary matrix of the 17 prostatectomy PCa cases showing interfocal heterogeneity regarding $E R G$ rearrangement status.

B: Summary matrix of the 5 cystoprostatectomy PCa cases showing interfocal heterogeneity regarding $E R G$ rearrangement status.

C: Summary matrix of the 10 TUR-P cases showing heterogeneity between different cores regarding $E R G$ rearrangement status.

\section{Figure 4:}

H\&E stained whole mount section of a prostate containing a PCa focus in the transition zone (outlined) harboring the ERG rearrangement.

* indicates the lumen of the prostatic urethra. 


\section{References:}

1. Scheble VJ, Braun M, Beroukhim R et al. ERG rearrangement is specific to prostate cancer and does not occur in any other common tumor. Mod Pathol 2010;23;1061-1067.

2. Tomlins SA, Bjartell A, Chinnaiyan AM et al. ETS gene fusions in prostate cancer: from discovery to daily clinical practice. Eur Urol 2009;56;275-286.

3. Guo CC, Zuo G, Cao D, Troncoso P, Czerniak BA. Prostate cancer of transition zone origin lacks TMPRSS2-ERG gene fusion. Mod Pathol $2009 ; 22 ; 866-871$.

4. Sieverding $M$, Matterne $U$, Ciccarello $L$, Luboldt $H$ J. [Early detection of prostate cancer in Germany. A study of a representative random sample of the population]. Urologe $A$ 2008;47;1233-1238.

5. Perner S, Svensson MA, Hossain RR et al. ERG Rearrangement Metastasis Patterns in Locally Advanced Prostate Cancer. Urology 2009.

6. Barry M, Perner S, Demichelis F, Rubin MA. TMPRSS2-ERG fusion heterogeneity in multifocal prostate cancer: clinical and biologic implications. Urology 2007;70;630-633.

7. Mehra R, Han B, Tomlins SA et al. Heterogeneity of TMPRSS2 Gene Rearrangements in Multifocal Prostate Adenocarcinoma: Molecular Evidence for an Independent Group of Diseases. Cancer Res 2007;67;7991-7995.

8. Furusato $B$, Gao $C L$, Ravindranath $L$ et al. Mapping of TMPRSS2-ERG fusions in the context of multi-focal prostate cancer. Mod Pathol $2008 ; 21 ; 67-75$.

9. Scheble VJ, Braun M, Wilbertz $T$ et al. ERG rearrangement in small cell prostatic and lung cancer. Histopathology 2010;56;937-943.

10. Tomlins SA, Rhodes DR, Perner $S$ et al. Recurrent fusion of TMPRSS2 and ETS transcription factor genes in prostate cancer. Science 2005;310;644-648.

11. Perner S, Demichelis F, Beroukhim R et al. TMPRSS2:ERG FusionAssociated Deletions Provide Insight into the Heterogeneity of Prostate Cancer. Cancer Res 2006;66;8337-8341.

12. Perner S, Mosquera JM, Demichelis $F$ et al. TMPRSS2-ERG fusion prostate cancer: an early molecular event associated with invasion. Am J Surg Pathol 2007;31;882-888.

13. Esgueva R, Perner S, C JL et al. Prevalence of TMPRSS2-ERG and SLC45A3-ERG gene fusions in a large prostatectomy cohort. Mod Pathol 2010;23;539-546.

14. Pflueger $D$, Rickman DS, Sboner $A$ et al. N-myc downstream regulated gene 1 (NDRG1) is fused to ERG in prostate cancer. Neoplasia 2009;11;804-811.

15. Mosquera JM, Mehra R, Regan MM et al. Prevalence of TMPRSS2ERG fusion prostate cancer among men undergoing prostate biopsy in the United States. Clin Cancer Res 2009;15;4706-4711. 
16. Demichelis $F$, Fall $K$, Perner $S$ et al. TMPRSS2:ERG gene fusion associated with lethal prostate cancer in a watchful waiting cohort. Oncogene 2007;26;4596-4599.

17. Attard G, Clark J, Ambroisine L et al. Duplication of the fusion of TMPRSS2 to ERG sequences identifies fatal human prostate cancer. Oncogene 2008;27;253-263.

18. Kumar-Sinha C, Tomlins SA, Chinnaiyan AM. Recurrent gene fusions in prostate cancer. Nat Rev Cancer 2008.

19. Mao X, Yu Y, Boyd LK et al. Distinct genomic alterations in prostate cancers in Chinese and Western populations suggest alternative pathways of prostate carcinogenesis. Cancer Res 2010;70;52075212.

20. Noel EE, Ragavan N, Walsh MJ et al. Differential gene expression in the peripheral zone compared to the transition zone of the human prostate gland. Prostate Cancer Prostatic Dis 2008;11;173-180.

21. King CR, Ferrari M, Brooks JD. Prognostic significance of prostate cancer originating from the transition zone. Urol Oncol 2009;27;592-597.

22. Sakai I, Harada K, Hara I, Eto H, Miyake H. A comparison of the biological features between prostate cancers arising in the transition and peripheral zones. BJU Int 2005;96;528-532.

23. Sakai I, Harada K, Kurahashi T, Yamanaka K, Hara I, Miyake H. Analysis of differences in clinicopathological features between prostate cancers located in the transition and peripheral zones. Int $J$ Urol 2006;13;368-372.

24. Greene DR, Wheeler TM. Clinical relevance of the individual prostate cancer focus. Cancer Invest 1994;12;425-437.

25. Greene DR, Wheeler TM, Egawa S, Dunn JK, Scardino PT. A comparison of the morphological features of cancer arising in the transition zone and in the peripheral zone of the prostate. $J$ Urol $1991 ; 146 ; 1069-1076$.

26. Noguchi M, Stamey TA, Neal JE, Yemoto CE. An analysis of 148 consecutive transition zone cancers: clinical and histological characteristics. J Urol 2000;163;1751-1755.

27. Shannon BA, McNeal JE, Cohen RJ. Transition zone carcinoma of the prostate gland: a common indolent tumour type that occasionally manifests aggressive behaviour. Pathology 2003;35;467-471.

28. Falzarano SM, Navas M, Simmerman $\mathrm{K}$ et al. ERG rearrangement is present in a subset of transition zone prostatic tumors. Mod Pathol 2010.

29. Mehra R, Tomlins SA, Shen R et al. Comprehensive assessment of TMPRSS2 and ETS family gene aberrations in clinically localized prostate cancer. Mod Pathol 2007; 20;538-544.

30. Rajput $A B$, Miller MA, De Luca $A$ et al. Frequency of the TMPRSS2:ERG gene fusion is increased in moderate to poorly differentiated prostate cancers. J Clin Pathol 2007;60;1238-1243.

31. Cheville JC, Karnes RJ, Therneau TM et al. Gene panel model predictive of outcome in men at high-risk of systemic progression 
and death from prostate cancer after radical retropubic prostatectomy. J Clin Oncol 2008;26;3930-3936.

32. Nam RK, Sugar L, Wang $Z$ et al. Expression of TMPRSS2:ERG gene fusion in prostate cancer cells is an important prognostic factor for cancer progression. Cancer Biol Ther 2007;6;40-45.

33. Nam RK, Sugar L, Yang $W$ et al. Expression of the TMPRSS2:ERG fusion gene predicts cancer recurrence after surgery for localised prostate cancer. Br J Cancer 2007;97;1690-1695.

34. Darnel AD, Lafargue CJ, Vollmer RT, Corcos J, Bismar TA. TMPRSS2ERG fusion is frequently observed in Gleason pattern 3 prostate cancer in a Canadian cohort. Cancer Biol Ther 2009;8;125-130.

35. Gopalan A, Leversha MA, Satagopan JM et al. TMPRSS2-ERG gene fusion is not associated with outcome in patients treated by prostatectomy. Cancer Res 2009;69;1400-1406.

36. Dai MJ, Chen LL, Zheng YB et al. [Frequency and transcript variant analysis of gene fusions between TMPRSS2 and ETS transcription factor genes in prostate cancer]. Zhonghua Yi Xue Za Zhi $2008 ; 88 ; 669-673$.

37. Rouzier C, Haudebourg J, Carpentier $X$ et al. Detection of the TMPRSS2-ETS fusion gene in prostate carcinomas: retrospective analysis of 55 formalin-fixed and paraffin-embedded samples with clinical data. Cancer Genet Cytogenet 2008;183;21-27.

38. Tu JJ, Rohan S, Kao J, Kitabayashi N, Mathew S, Chen YT. Gene fusions between TMPRSS2 and ETS family genes in prostate cancer: frequency and transcript variant analysis by RT-PCR and FISH on paraffin-embedded tissues. Mod Pathol 2007;20;921-928.

39. Yoshimoto M, Joshua AM, Chilton-Macneill $S$ et al. Three-Color FISH Analysis of TMPRSS2/ERG Fusions in Prostate Cancer Indicates That Genomic Microdeletion of Chromosome 21 Is Associated with Rearrangement. Neoplasia 2006;8;465-469.

40. Lapointe J, Kim YH, Miller MA et al. A variant TMPRSS2 isoform and ERG fusion product in prostate cancer with implications for molecular diagnosis. Mod Pathol 2007;20;467-473. 


\section{Figure 1}

A
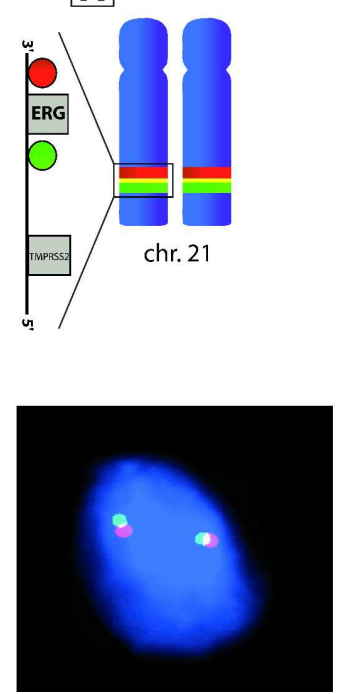

B
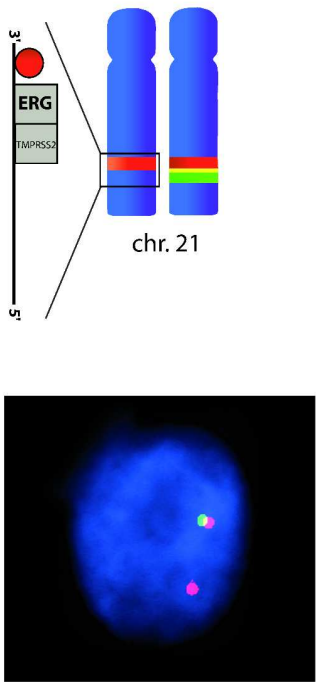

C
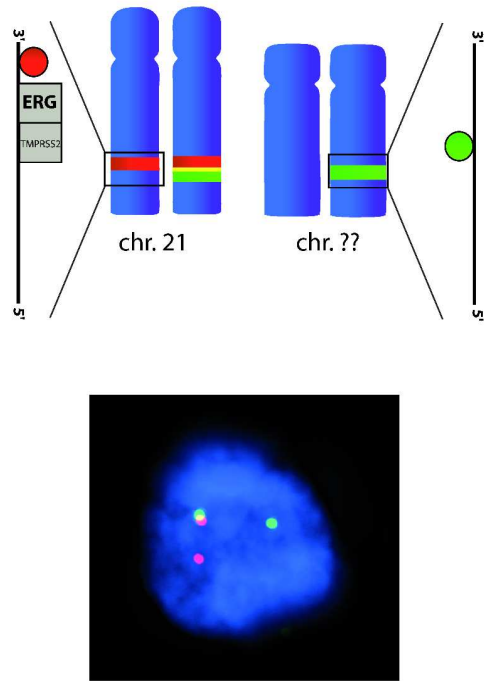

Figure 1:

Detection of the ERG rearrangement.

Schematic displaying the dual colour probe ERG break-apart Fluorescence in-situ Hybridization (FISH) assay as used for the indirect detection of ERG Rearrangements. Red-labelled centromeric and the green-labelled telomeric probes span the ERG locus on chromosomes 21. If a break-apart occurs, the green signal is either lost (ERG rearrangement through deletion) or translocated (ERG rearrangement through insertion). An ERG break-apart as determined by FISH accounts for a fusion of ERG with TMPRSS2, SLC45A or NDRG.

A: Wild type ERG rearrangement status. Metaphase chromosomes 21 with dual colour probes spanning the centromeric and telomeric region of the ERG locus. Interphase nucleus of a peripheral zone prostate cancer cell (below) shows one pairs of adjacent red and green signals with yellow signal overlapping, depicting a wild type ERG rearrangement status.

B: ERG rearrangement through deletion. One chromosome 21 lacks the telomeric probe spanning the ERG locus. Interphase nucleus of a transitional zone prostate cancer cell (below) shows two adjacent red and green signals, as well as one single red signal, depicting a heterozygous ERG rearrangement through deletion.

C: ERG rearrangement through insertion. The $5^{\prime}$ telomeric ERG-spanning probe of one chromosome

21 is translocated to another chromosome. Interphase nucleus of a peripheral zone prostate cancer cell (below) shows two adjacent red and green signals, as well as one separate red and green signal, depicting a heterozygous ERG rearrangement through insertion.

$287 \times 181 \mathrm{~mm}(550 \times 550 \mathrm{DPI})$ 
Figure 2

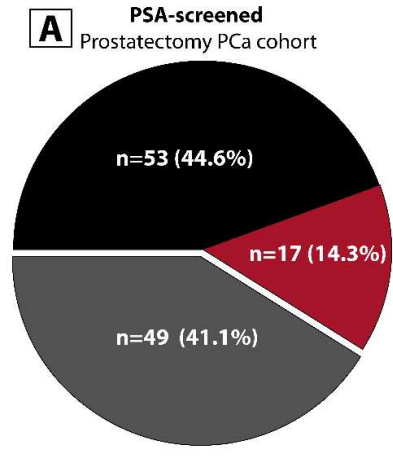

$\mathbf{5 9 \%}$ of cases $E R G$ rearranged $49 \%$ of tumour foci $E R G$ rearranged

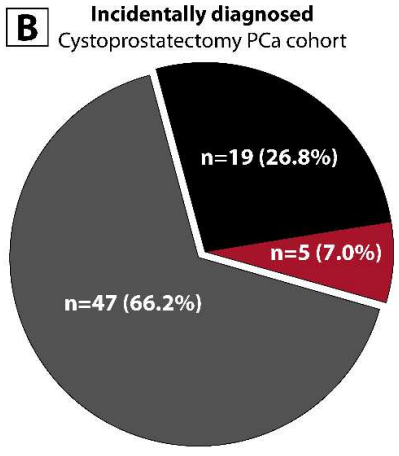

$\mathbf{3 4 \%}$ of cases $E R G$ rearranged $\mathbf{2 9} \%$ of tumour foci $E R G$ rearranged

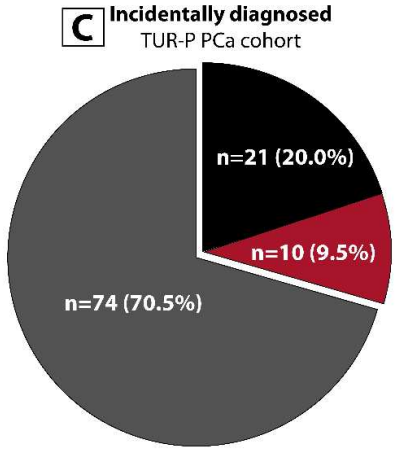

$\mathbf{3 0} \%$ of cases $E R G$ rearranged

Color coding for ERG rearrangement status

\begin{tabular}{|c|}
\hline Color coding for ERG rearrangement status \\
Rearrangement \\
Interfocal heterogeneity \\
\hline No rearrangement \\
\hline
\end{tabular}

Figure 2:

ERG rearrangement prevalence of two incidentally diagnosed PCa cohorts and one PSA-screened prostatectomy cohort. Each core/focus of an individual case was independently assessed for the ERG rearrangement status.

A: Pie chart displaying the ERG rearrangement status of the prostatectomy PCa cohort.

B: Pie chart displaying the ERG rearrangement status of the cystoprostatectomy PCa cohort.

C: Pie chart displaying the ERG rearrangement status of the TUR-P cohort.

$295 \times 185 \mathrm{~mm}(550 \times 550 \mathrm{DPI})$ 


\section{Figure 3}

Interfocal heterogeneity in prostatectomy PCa cases

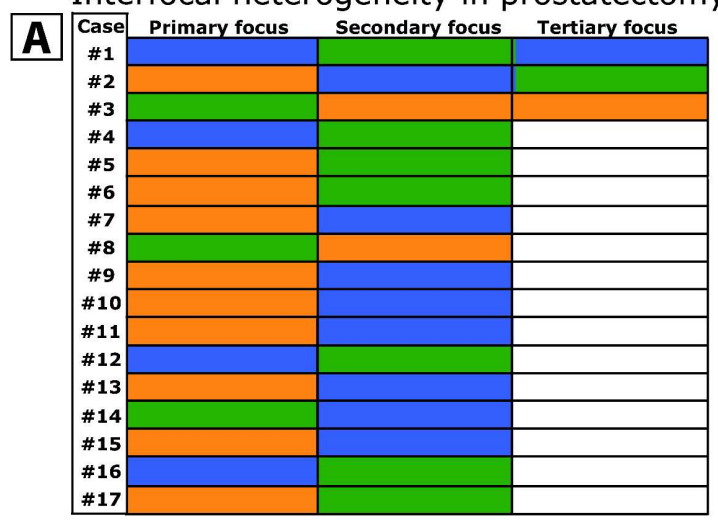

Interfocal heterogeneity in cystoprostatectomy PCa cases

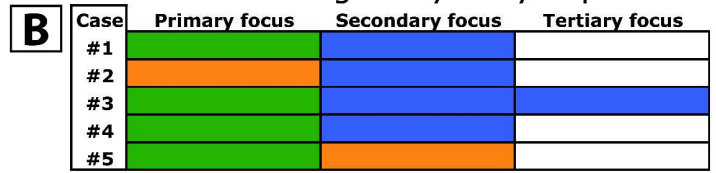

Heterogeneous TUR-P PCa cases
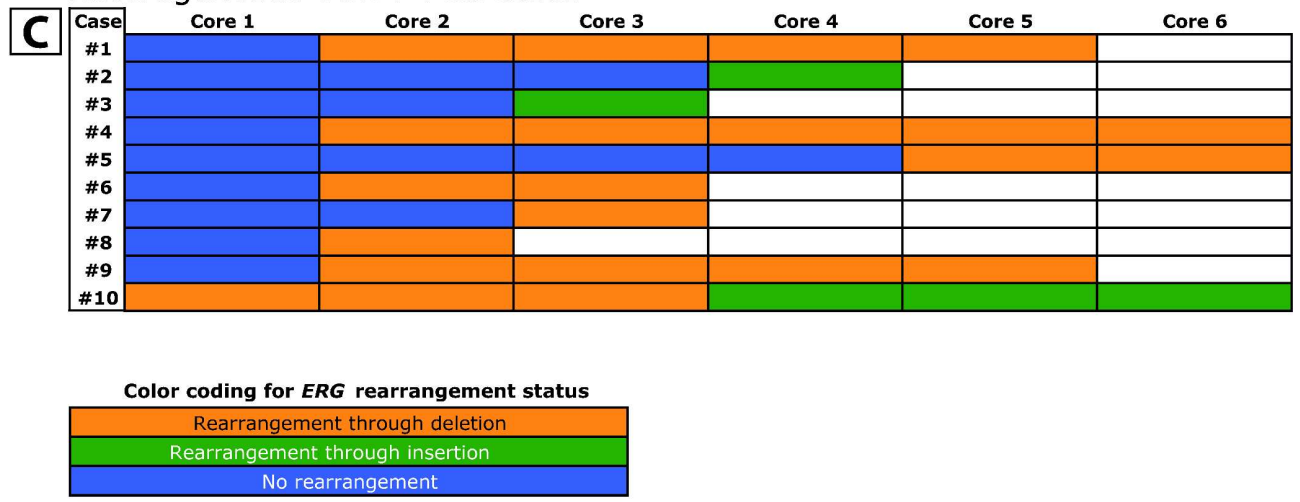

Figure 3:

ERG rearrangement heterogeneity of two incidentally diagnosed PCa cohorts and one PSA-screened prostatectomy cohort. Each core/focus of an individual case was independently assessed for the ERG rearrangement status.

A: Summary matrix of the 17 prostatectomy PCa cases showing interfocal heterogeneity regarding ERG rearrangement status.

B: Summary matrix of the 5 cystoprostatectomy PCa cases showing interfocal heterogeneity regarding ERG rearrangement status.

C: Summary matrix of the 10 TUR-P cases showing heterogeneity between different cores regarding ERG rearrangement status. 
$191 \times 232 \mathrm{~mm}(550 \times 550 \mathrm{DPI})$ 


\section{Figure 4}

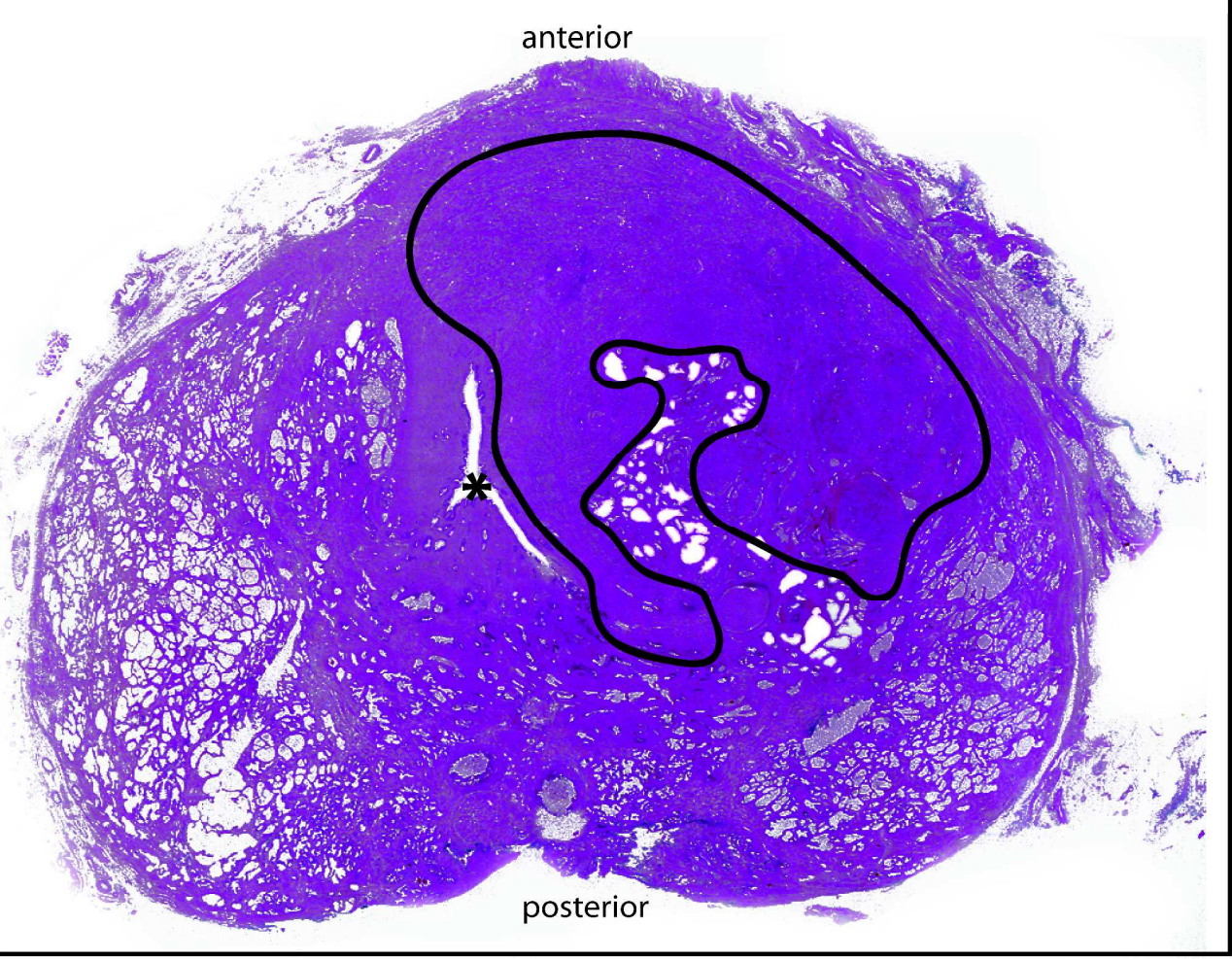

.Figure 4:

H\&E stained whole mount section of a prostate containing a PCa focus in the transition zone (outlined) harboring the ERG rearrangement.

* indicates the lumen of the prostatic urethra.

$$
192 \times 155 \mathrm{~mm}(550 \times 550 \text { DPI) }
$$




\section{Table 1}

\begin{tabular}{|c|c|c|c|}
\hline & $\begin{array}{c}\begin{array}{c}\text { Prostatectomy } \\
\text { cohort }\end{array} \\
\text { (PSA-screened) } \\
\\
66 \text { years } \pm 6.2\end{array}$ & $\begin{array}{c}\text { Cystoprostatectomy } \\
\text { cohort } \\
\text { (incidentally } \\
\text { diagnosed) } \\
68.5 \text { years } \pm 7.3\end{array}$ & $\begin{array}{l}\text { TUR-P cohort } \\
\text { (incidentally } \\
\text { diagnosed) } \\
72 \text { years } \pm 8.2\end{array}$ \\
\hline & $\% E R G$ rearranged & $\% E R G$ rearranged & $\% E R G$ rearranged \\
\hline Total number of cases & $58.9 \%(70$ of 119$)$ & $33.8 \%(24$ of 71$)$ & $29.5 \%(31$ of 105$)$ \\
\hline Total number of tumour foci & $49.4 \%$ (77 of 156$)$ & $29.5 \%(28$ of 95$)$ & N.A. \\
\hline PZ tumour foci & $51.7 \%(75$ of 145$)$ & $30.2 \%(26$ of 86$)$ & N.A. \\
\hline TZ tumour foci & $18.1 \%(2$ of 11$)$ & $22.2 \%(2$ of 9$)$ & N.A. \\
\hline Primary tumour foci & $56.9 \%(62$ of 109$)$ & $34.8 \%(24$ of 69$)$ & N.A. \\
\hline Secondary tumour foci & $31.7 \%(13$ of 41$)$ & $19.0 \%(4$ of 21$)$ & N.A. \\
\hline Tertiary tumour foci & $33.3 \%(2$ of 6$)$ & $20.0 \%(1$ of 5$)$ & N.A. \\
\hline Combined Gleason score (over all foci) $\leq 6$ & $64.2 \%(9$ of 14$)$ & $33.3 \%(12$ of 36$)$ & $18.9 \%(7$ of 37$)$ \\
\hline Combined Gleason score (over all foci) $=7$ & $62.5 \%(50$ of 80$)$ & $44.0 \%(11$ of 25$)$ & $19.0 \%(4$ of 21$)$ \\
\hline Combined Gleason score (over all foci) $\geq 8$ & $44.0 \%(11$ of 25$)$ & $10 \%(1$ of 10$)$ & $42.5 \%(20$ of 47$)$ \\
\hline
\end{tabular}

PZ: Peripheral zone

TZ: Transition zone

N.A.: Not applicable 


\section{Colour Work Agreement Form}

Form No: SN_Upw2000_P_CoW_F2

If you have any problems or queries please contact the Production Editor for guidance (details can be found in the Instructions for Authors)

If you have submitted a manuscript that contains colour figures, it is the policy of the journal for authors to meet the full cost of colour reproduction. Blackwell Publishing require you to complete this form. Please fill in your contact details and then complete the table as instructed. Note: this form must be filled in for all manuscripts that have been submitted with colour work, even if you subsequently choose not to publish either in print or online in colour.

We are unable to process your manuscript until we receive your instructions so please return this form as quickly as possible!

Journal name: Histopathology

Corresponding Author: Sven Perner

Manuscript No/Title: HISTOP-06-10-0305.R2 / Relevance of cohort design for studying the prevalence of the ERG rearrangement in prostate cancer

Your Name: Sven Perner

Address: Universitätsklinikum Bonn, Institut f. Pathologie, Sigmund-Freud-Str. 25, 53127 Bonn

Fax:

Tel: $\quad+4915158233448$

e-mail: sven.perner1972@gmail.com

WHAT YOU NEED TO DO

Please complete the table on page 2, placing an ' $X$ ' in the relevant column for each figure.

The costs are shown at the top of each column. Please calculate the total cost for each

category at the foot of the table, and then add VAT if applicable (see below).

All customers MUST quote their VAT numbers

Customers in the UK: $\quad$ add VAT at $17.5 \%$.

Customers elsewhere in the EU:

- Customers registered for VAT: No VAT (please complete details below)

VAT registration number:

Name of organisation:

- Customers NOT registered for VAT: Add VAT at $17.5 \%$.

Customers outside the EU: $\quad$ No VAT

PAGE 1

\begin{tabular}{|c|c|c|c|c|}
\hline \multicolumn{5}{|c|}{$<<<$ For internal use ONLY $>>>$} \\
\hline JPCMS No & Journal acronym & Volume & Issue & Print run \\
\hline
\end{tabular}


Please now complete this table (Remember: only one ' $X$ ' per figure).

\begin{tabular}{|l|l|l|l|}
\hline Figure Number & $\begin{array}{l}\text { Column 1 } \\
\text { Colour in print and online* }\end{array}$ & $\begin{array}{l}\text { Column 2 } \\
\text { Colour online ONLY }\end{array} \quad \begin{array}{l}\text { Column 3 } \\
\text { Black white in print and online }\end{array}$ \\
\hline Costs & $\begin{array}{l}\text { 1st and 2nd figures = Free } \\
\text { Subsequent figures }=£ 60 \text { each }\end{array}$ & $£ 50$ each & Free \\
\hline 1 & $\mathrm{x}$ & & \\
\hline 2 & $\mathrm{x}$ & & \\
\hline 3 & $\mathrm{x}$ & & \\
\hline 4 & $\mathrm{X}$ & & \\
\hline 5 & & & \\
\hline 6 & & & \\
\hline 7 & & & \\
\hline 8 & & & \\
\hline 9 & & & \\
\hline 10 & & & \\
\hline $\begin{array}{l}\text { Total number of } \\
\text { figures of each type }\end{array}$ & & & \\
\hline
\end{tabular}

\begin{tabular}{|ll|ll|}
\hline \multicolumn{4}{|c|}{ 'Quick cost lookup table for colour figures in print and online: } \\
\hline No. of colour figs & Cost & No. of colour figs & Cost \\
\hline 1 & Free & 6 & $£ 240$ \\
2 & Free & 7 & $£ 300$ \\
3 & $£ 60$ & 8 & $£ 360$ \\
4 & $£ 120$ & 9 & $£ 420$ \\
5 & $£ 180$ & 10 & $£ 480$ \\
\hline
\end{tabular}

Column 1 total $£$

Column 2 total $£$

VAT (if applicable) $£ \quad 21$

Total: $£ \quad 141$

Payment by Credit Card (MasterCard/VISA/American Express)

Blackwell Publishing accepts payment in three major currencies:

Pounds Sterling, United States Dollars and the Euro. Please indicate which currency you would like to pay in:

Pounds Sterling $(£) \square /$ United States Dollars* (US\$) $\square /$ Euros $^{*}(€)$ X (tick one)

${ }^{*}$ Conversions from US $\$$ or $€$ will be made at our monthly rate.

Please debit my credit card number: \begin{tabular}{|l|l|l|l|l|l|l|l|l|l|l|l|l|l|l|l|}
\hline 5 & 3 & 1 & 0 & 0 & 2 & 3 & 0 & 1 & 8 & 2 & 8 & 2 & 2 & 0 & 8 \\
\hline
\end{tabular}

Verification ID Number: \begin{tabular}{|l|l|l|l|}
\hline & 2 & 6 & 2 \\
\hline
\end{tabular}

Name on card _ Dr. Sven Roger Perner

Expiry Date $\quad 02 \quad / \quad 2013$

With the sum of $£ 141$

Signature _ Sven Perner

Date 20th Oct 2010

Note: payment by credit card can only be accepted if all sections are completed.

Please use BLOCK CAPITALS where possible.

Payment by Purchase Order

If you are asking your university or institution to pay we require purchase order details

I attach order number dated

Please now return all pages of your completed form as a scanned electronic file (preferred option), or by fax or post to the production editor for your journal.

Contact details can be found in the instructions for authors.

Published on behalf of the British Division of the International Academy of Pathology 